\title{
REVISED The benefits of mindfulness in mental healthcare
}

\section{professionals [version 2; peer review: 2 approved]}

\section{Tayler Watson (D1) Owen Walker2, Robin Cann (D)3, Ashwin K Varghese (D2)}

\author{
${ }^{1}$ Mental Health, Drugs and Alcohol Service, Barwon Health, Geelong, Victoria, 3220, Australia \\ ${ }^{2}$ Department of Psychiatry, Monash Health, Melbourne, Victoria, 3806, Australia \\ ${ }^{3}$ Nova Scotia Health Authority, Halifax, Nova Scotia, NS B3S OH6, Canada
}

\begin{tabular}{l}
\hline V2 First published: 25 Oct 2021, 10:1085 \\
https://doi.org/10.12688/f1000research.73729.1 \\
Latest published: 31 Jan 2022, 10:1085 \\
https://doi.org/10.12688/f1000research.73729.2 \\
\hline
\end{tabular}

\section{Abstract}

Background: Burnout is a widely reported syndrome consisting of emotional exhaustion, depersonalization, and a lowered sense of accomplishment. Mindfulness practices have been shown to be useful in lowering distress and burnout in clinical and non-clinical cohorts. Our aim was to explore the potential personal and occupational benefits of a structured mindfulness intervention on a cohort of mental health professionals. A mixed-methods approach was utilised in order to enhance the exploratory power of the study. Methods: We conducted a pilot study involving healthcare practitioners employed at a community outpatient mental health clinic. As a pilot, we relied on a single group and implemented a quasi-experimental, simultaneous mixed methods design by incorporating both quantitative pre- and post- testing alongside written qualitative post-test responses.

Results: Analysis of the data demonstrated a significant difference between overall mindfulness when comparing post-test (mean=140.8, standard deviation=18.9) with pre-test data (mean=128.3, standard deviation=28.6). Participants also showed a statistically significant difference in three of the subscales: observation, describing, and nonreactivity. A moderate effect size was seen for each of the above differences. Analysis of the qualitative data revealed a range of potential themes which may be used to explain the differences exhibited across participants' personal and professional lives, which can be grouped into two thematic overarching groups: emotional reactivity and listening/communicating. Conclusions: The results of this pilot study indicate that a structured, six-week mindfulness program has the potential to benefit clinicians, personally by reducing emotional reactivity and professionally by promoting deep listening and communication.

\section{Keywords}

Mindfulness, Mental Health, Burnout, Psychiatry

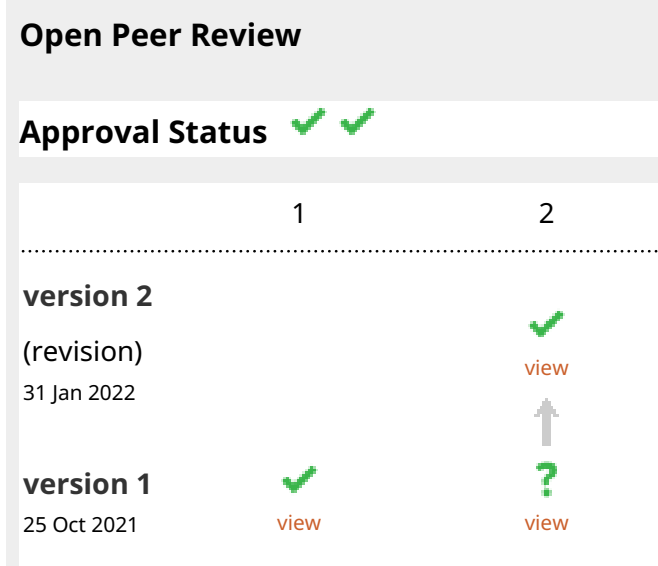

1. Dr. Richard Mottershead ID, RAK Medical and Health Sciences University, Ras Al Khaimah, United Arab Emirates

2. Veena S. Singaram (D), University of KwaZulu-Natal, Durban, South Africa Any reports and responses or comments on the article can be found at the end of the article. 
Corresponding author: Ashwin K Varghese (ashwinvarghese@yahoo.ca)

Author roles: Watson T: Formal Analysis, Investigation, Methodology, Project Administration, Supervision, Validation, Writing - Original Draft Preparation, Writing - Review \& Editing; Walker O: Formal Analysis, Methodology, Project Administration, Software, Supervision, Writing - Original Draft Preparation, Writing - Review \& Editing; Cann R: Data Curation, Formal Analysis, Methodology, Writing - Original Draft Preparation, Writing - Review \& Editing; Varghese AK: Conceptualization, Data Curation, Formal Analysis, Investigation, Methodology, Project Administration, Resources, Supervision, Validation, Writing - Original Draft Preparation, Writing - Review \& Editing Competing interests: No competing interests were disclosed.

Grant information: The author(s) declared that no grants were involved in supporting this work.

Copyright: $\odot 2022$ Watson T et al. This is an open access article distributed under the terms of the Creative Commons Attribution License, which permits unrestricted use, distribution, and reproduction in any medium, provided the original work is properly cited.

How to cite this article: Watson T, Walker $\mathrm{O}$, Cann $\mathrm{R}$ and Varghese AK. The benefits of mindfulness in mental healthcare professionals [version 2; peer review: 2 approved] F1000Research 2022, 10:1085 https://doi.org/10.12688/f1000research.73729.2

First published: 25 Oct 2021, 10:1085 https://doi.org/10.12688/f1000research.73729.1 


\section{REVISED Amendments from Version 1}

Changes in this revision include: addition of linking statement and evidence for relationship between perceived stress and burnout in paragraph one of introduction and paragraph two under sub-heading 'questionnaires'; addition of details in data analysis (paragraph one, under subheading 'data analysis'); addition of discussion on impacts of COVID-19 on study's relevance (paragraph four, under heading 'discussion').

Any further responses from the reviewers can be found at the end of the article

\section{Introduction}

Proposed by Maslach et al. in 1986, burnout is now widely accepted as consisting of emotional exhaustion, depersonalization and a lowered sense of accomplishment, contributing to an overall reduction in psychological wellbeing. ${ }^{1}$ High levels of burnout have been recorded across industries ${ }^{2-4}$ with common etiological themes related to workload demands, low occupational satisfaction and psychosocial stressors. ${ }^{5-7}$ Some reports suggest up to $80 \%$ of physicians meet criteria for burnout, ${ }^{8}$ however more conservative estimates suggest around half of physicians experience burnout. ${ }^{9}$ Up to $78 \%$ of psychiatrists and $21 \%$ of psychiatry residents are experiencing burnout at any one time. ${ }^{10,11}$ Around half of family physicians report high levels of emotional exhaustion and depersonalization, and more than two thirds of young oncologists report burnout. ${ }^{12,13}$ Two thirds of mental health clinicians including psychologists, social workers and nurses, also report distress. ${ }^{14}$ Perceived stress is positively correlated with burnout. ${ }^{15}$ Furthermore, Maslach's dimensions of depersonalisation and lowered sense of accomplishment predict perceived stress levels. ${ }^{15}$ Burnout is associated with major depression, poor patient outcomes and more negative feelings towards patients. ${ }^{16-18}$ Physician burnout also results in significant economic loss. ${ }^{19}$ By contrast, psychiatry is well placed to drive positive change. ${ }^{20}$ It is clear that burnout is a key issue facing the medical and psychosocial professions. While the existence of the phenomenon is well understood, attempts to rectify the problem remain scarce. Interventions would need to be easily administered and suitably scalable. Mindfulness represents a promising avenue for exploration, given the ease with which it can be taught and maintained.

Mindfulness can be defined as "paying attention to present moment experiences with openness and curiosity and a willingness to be with what is" (UCLA health). Operational definitions distinguish two components of mindfulness: self-regulation of attention and orientation to the present moment characterized by curiosity, openness and acceptance. ${ }^{21}$ A range of mindfulness practices and techniques have been developed and applied for use in both mental and physical illnesses. $^{22}$ One of these practices, Mindfulness Based Stress Reduction (MBSR), demonstrates efficacy in reducing stress, depression, anxiety and negative ruminations. ${ }^{23-25}$ MBSR delivered to therapists in training enhanced selfcompassion and positive affect. ${ }^{26}$ When applied to physicians, MBSR may reduce burnout, enhance resilience and improve patient care. ${ }^{27-29}$ Mindfulness has also been correlated with neurophysiological and neurobiological changes in key brain regions associated with the observed phenomenology. ${ }^{30}$

This preliminary pilot study aims to explore the benefits of a mindfulness intervention on mental health practitioners. Mixed methods approaches have been shown to be an effective way to allow for a more robust exploration of a phenomenon than each individual method used in isolation. ${ }^{31}$ Collecting both types of data can greatly assist in the exploratory nature of the study, with qualitative information used to further explore, as well as potentially explain, the quantitative analysis.

\section{Methods}

Study design

This pilot study employed a single group quasi-experimental, simultaneous mixed-methods design by utilizing quantitative pre and post testing paired with a written qualitative post-assessment. The qualitative component of this study used a descriptive phenomenological method.

\section{Participants and recruitment}

13 healthcare practitioners employed at a community outpatient mental health clinic in Amherst, Nova Scotia were recruited for the study in October 2016 (Table 1). Recruitment was completed via email and word-of-mouth discussion. Interested clinicians were then asked to refer colleagues who were not reached via email. Non-probability convenience sampling was used to recruit participants with desired study characteristics and expeditiously collect qualitative data. Snowball sampling increased the potential number of participants. This number of participants was chosen as evidence suggests theoretical saturation occurs at 12 respondents. ${ }^{32,33}$ 
Table 1. Displays the total number and percentage of participants $(n=13)$ by role and gender.

\begin{tabular}{|l|l|l|}
\hline Clinical role & Psychiatrists & $6(46 \%)$ \\
\hline & Social Workers & $6(46 \%)$ \\
\hline \multirow{2}{*}{ Gender } & Family Physician & $1(7 \%)$ \\
\hline & Male & $4(31 \%)$ \\
\hline & Female & $9(69 \%)$ \\
\hline
\end{tabular}

\section{Mindfulness program}

The Mindfulness Awareness Practices (MAPs) program is a six-week, validated protocol delivered by a trained instructor. The intervention has demonstrated usefulness in reducing distress in clinical populations. ${ }^{34,35}$ Participants completed the MAPs six-week course, in November and December 2016. Week one included a two-hour workshop focussed on a conceptual introduction to mindfulness and psychoeducation. In weeks two to five, participants engaged in weekly didactic and experiential 90-minute workshops learning a range of mindfulness techniques, such as sitting, relational and movement meditation and skills for recognising positive and difficult emotions. Participants were encouraged to complete and record 'homework' activities, including guided meditation practice. The program was delivered on-site at an outpatient community mental health clinic during the participants' regular workday.

\section{Questionnaires}

The Five Facet Mindfulness Questionnaire (FFMQ), first proposed in 2006, is a robust, 39-item self-report, Likert type scale measuring five correlates of mindfulness; observing, describing, acting with awareness, non-judging and non-reacting. ${ }^{36,37}$ The survey's factor structure is available in the original article. ${ }^{36}$ The scale exhibits reliable construct validity across facets. ${ }^{38}$ Examples of statements include "I'm good at finding words for my feelings" and "I watch my feelings without getting lost in them". Respondents are then asked to rate these statements with five potential options (never or very rarely true, rarely true, sometimes true, often true, very often or always true).

The Perceived Stress Scale (PSS) ${ }^{10}$ is an extensively used self-report questionnaire designed to measure individual stress, demonstrating high internal consistency across populations $(\alpha=0.82) .{ }^{39,40}$ The PSS correlates with burnout, as measured on the Maslach Burnout Inventory (MBI). ${ }^{15}$ In particular, the PSS is positively correlated with the dimensions of depersonalisation and emotional exhaustion. Furthermore, lowered personal accomplishment and depersonalisation predict levels of perceived stress. ${ }^{15}$ The PSS was used due to ease of administration and established reliability. Participants were asked 10 questions, scored on a Likert scale (never, almost never, sometimes, fairly often or very often). Questions assess the level of perceived stress one has experienced within the last month. For example, "in the last month, how often have you been upset because of something that happened unexpectedly?". The scale is widely available and has been validated in several languages. ${ }^{41-43}$

\section{Data collection}

Prior to the initial session in week one participants completed handwritten, hardcopy FFMQ and PSS questionnaires. These questionnaires were then collected by the principal investigator and securely stored. Participants then completed six weeks of the intervention as described. Following the final session in week six, participants completed handwritten FFMQ and PSS questionnaires and a brief qualitative questionnaire as described below. The completed quantitative and qualitative questionnaires were then collected by the principal investigator and stored securely for analysis.

Qualitative data was obtained using brief, open-ended written survey questions that allowed for narrative-style feedback from participants, only completed post-intervention. The questions were developed to allow for rich and open responses in the context of the study design and intervention, and therefore were not based on pre-existing models or surveys. The questions were as follows:

"In what way has the six-week mindfulness training influenced your personal life?"

"In what way has the six-week mindfulness training influenced your clinical practice?"

Statistical analysis

FFMQ and PSS scores were set as primary outcome measures. Continuous data from the quantitative questionnaires was entered into SPSS (version 24). Paired samples t-tests were then conducted. Statistical significance was assumed at $0.05 \%$. Corresponding Cohen's D values were determined, and effect sizes established. Outcome variables included 
overall mindfulness, perceived stress and the five FFMQ subscales. Secondary outcome measures sought to explore the relationship between engagement and change in outcome scores, however statistical analysis was impacted by poor response rates.

Data analysis

Participants were provided with the two questions stated above, and in turn provided the researchers with handwritten responses. Written responses were transcribed to text using Microsoft Word (version 16.53) and some minor substitutions (e.g., replacing shorthand with full words) were made to aid legibility, with no impact to the meaning of the responses. $1 \%$ of words were illegible, despite consulting a second reviewer. Thematic analysis according to Braun and Clarke ${ }^{44}$ was $^{2}$ applied to the qualitative data to observe themes that arose across participants' varying experiences of the program. Investigators familiarized themselves with the data contained within the qualitative questionnaires. A manual semantic coding process was then applied to the dataset. Next, the authors applied pattern-based analysis to identify recurring themes and subthemes based on the frequency of codes. Themes and sub-themes were finalised and discussed in the context of the research question. This process allowed the authors to identify, analyse and describe recurrent themes and concepts observed within the dataset. Thematic analysis is a flexible approach that provides a framework for a rich discussion. ${ }^{45}$ Considering the subjectivity of the research topic, the authors adopted this approach to best reflect the true experience of the participants, and thus enhance trustworthiness.

\section{Reflexive statement}

The researchers acknowledge their own characteristics in the interpretation of the qualitative data. Interpreting authors were predominantly male from medical backgrounds with special interests in the subject matter, which we recognise may have impacted objectivity.

\section{Ethics and consent}

The Nova Scotia Health Authority Research Ethics Board granted an exemption from ethics approval as the study is a quality improvement/educational initiative. Verbal, informed consent was obtained from participants prior to commencement for use and publication of study data as all participants were staff of the health authority. The paper forms were stored in a locked cabinet and digital data were stored on password-protected computers.

\section{Results}

Primary outcome analysis

Demographic characteristics of the cohort are displayed in Table 1. Most participants were female and either worked in psychiatry or social work. Table 2 displays statistically significant results of paired sample T-tests, used to measure prepost change in overall mindfulness and FFMQ subscales. On the FFMQ, there was a statistically significant difference in overall mindfulness after participation in MAPs when compared to before participation $(\mathrm{p}=0.007)$ (Figure 1). Participants also demonstrated statistically significant changes on three subscales of the FFMQ: observation, describing and non-reactivity (Figure 2). There was a statistically significant increase in participants' ability to observe internal and external experiences when compared to baseline $(\mathrm{p}=0.036)$. Similarly, a statistically significant increase was also demonstrated in participants' ability to describe and label internal experiences after participation in MAPs than at the time of pre-test $(p=0.042)$. Finally, participants showed reduced reactivity to internal experiences and enhanced ability to allow the free flow of thought without rejection after participation in the study when compared to baseline $(p=0.019)$. Moderate effect sizes were demonstrated in the overall FFMQ (Cohen's $d=0.588$ ), observing (Cohen's $d=0.452$ ), describing (Cohen's $d=0.407$ ) and non-reacting (Cohen's $d=0.693$ ) subscales. The mean number of absences to sessions was 1.2. Reason for absences was not recorded. Three participants missed two or more sessions in total. No statistically significant difference in FFMQ scores was observed in this group $(\mathrm{p}=0.98)$. There were no statistically significant changes on the final two subscales of the FFMQ, awareness and non-judging; as well, no statistically significant changes in the perceived stress scale were observed.

Table 2. Displays mean change of FFMQ scales pre and post participation in MAPs, displayed with corresponding $\mathbf{t}$ and $\mathbf{p}$ values. Corresponding effect sizes presented as Cohen's $d$ values.

\begin{tabular}{|l|l|l|l|l|}
\hline & Mean change & $\mathbf{t}=$ & $\mathbf{p}=$ & Cohen's \\
\hline FFMQ overall & 12.6 & 3.2 & 0.007 & 0.59 \\
\hline FFMQ observing subscale & 2.54 & 2.37 & 0.036 & 0.45 \\
\hline FFMQ describing subscale & 2.38 & 2.28 & 0.042 & 0.41 \\
\hline FFMQ non-reacting subscale & 2.61 & 2.71 & 0.019 & 0.69 \\
\hline
\end{tabular}




\section{Overall Change in Five Facet Mindfulness Questionnaire Scores Pre \& Post Participation in MAPs}

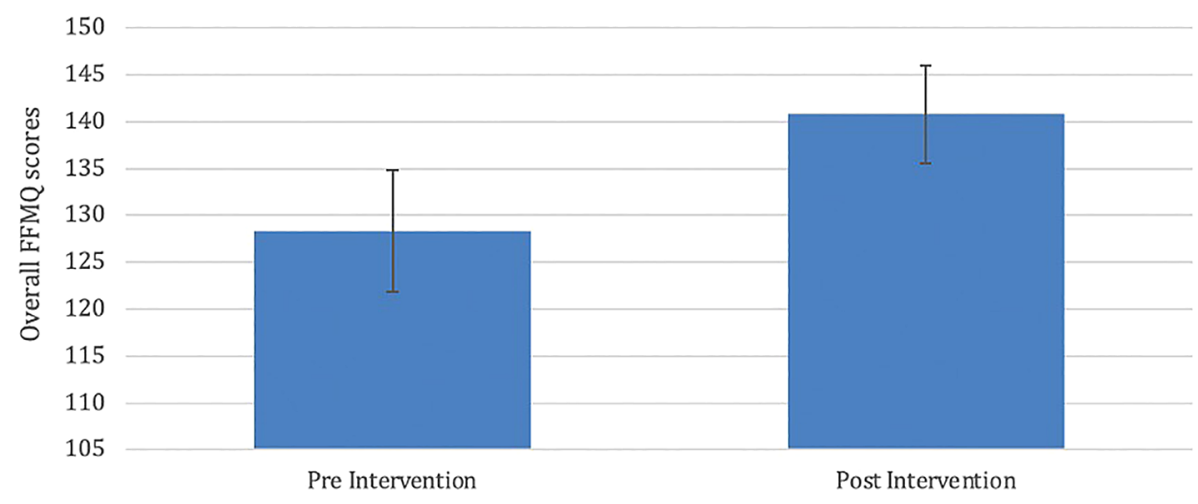

Figure 1. Mean change in overall FFMQ scores pre and post participation in MAPs protocol $(p=0.007)$. Error bars represent standard errors (SEM). FFMQ: Five Facet Mindfulness Questionnaire. MAPs: Mindfulness Awareness Practices.

Five Facet Mindfulness Questionnaire Subscale Scores Pre and Post Participation in MAPs

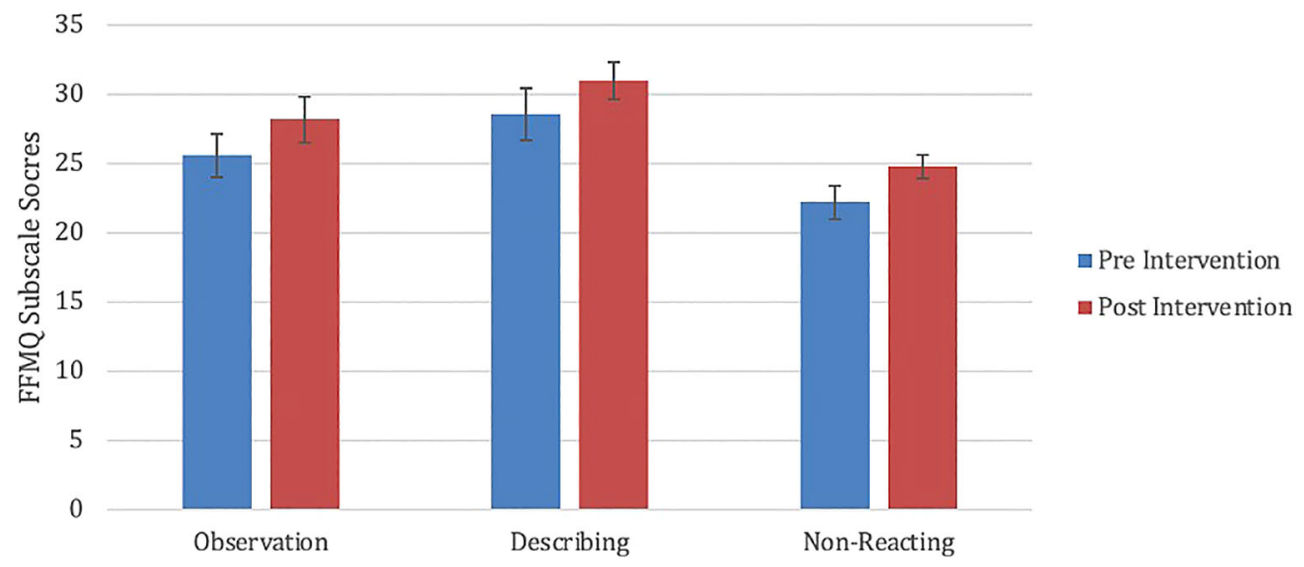

Figure 2. Mean change in observation $(p=0.036)$, describing $(p=0.042)$ and non-reacting $(p=0.019)$ FFMQ subscale scores following participation in MAPs. Error bars represent standard errors (SEM). FFMQ: Five Facet Mindfulness Questionnaire. MAPs: Mindfulness Awareness Practices.

\section{Qualitative analysis}

The responses for each of the two questions were initially analysed separately, with a range of sub-themes identified. Positive effects in participants' personal life and clinical practice were noted.

\section{Personal benefits}

A common response amongst participants was the change in their relationship with their emotions, particularly in their capacity to identify, observe, and subsequently regulate them, as evidenced by the following examples: "Better able to notice emotions", "Increased emotional regulation", "Checking in more often with feelings and sensations", "making room and allowing", "awareness of what I am experiencing in the moment", "I am less emotionally reactive", "I am more aware of my emotions and in better control of them".

Tolerance of uncertainty was another common benefit that arose. Some participants indicated that the intervention led to a decrease in their intolerance of uncertainty, a psychological phenomenon that is implicated in anxiety and related to one's ability to productively and functionally appraise unknown situations. Responses included: "Helped with decreasing attachment to things I can't control", "Tend to have a greater sense of agency with challenging life situations", "... using STOP and RAIN to pause, step back, identify, make space has definitely help me to cope better with uncertainty". Many 
participants commented on the benefits of the practical elements of the workshop and the subsequent increase in their therapeutic skillset, including the STOP and RAIN mnemonics (tools to remember how to navigate stressful situations).

Participants of this study indicated that having a dedicated space for self-reflection, particularly among a safe and supportive group, encouraged them to engage in more self-love which related to a theme of self-compassion. Responses included: "Given myself permission for self-care ...", "More loving kindness to myself ...", "Being more loving/ accepting of myself".

Some participants seemed able to translate the increase in self-care into motivation to improve their regular routine. Responses included: "It has rekindled my interest in mindful practice. Not only doing the homework but doing reading", "It has brought me back to regular practice which I'm thankful for", "Awareness of the importance of committing to a regular daily practice.", "Accountability for practice = more structured practice", "Practice self-compassion more frequently".

Responses included: "Using "STOP' fairly often”, "Gave me 'tool' to use with my child. Re-affirmed some positive skills I use/have already with 'data' (i.e., name emotion, loving kindness, touch.)", "Usefulness of STOP and RAIN - practical techniques ... using STOP and RAIN to pause, step back, identify, make space has definitely help me to cope better ...", "Gave me skills for relaxation when I am stressed.", "I particularly enjoyed the "what brings you joy" exercise and found myself asking my loved ones that question".

Finally, participants frequently discussed the positive impact that the above factors had on their interpersonal relationships: Responses included: "It has improved my relationships with my friends and family. I am less emotionally reactive with my spouse and children”, "I'm more present in my relationships”, “... more loving ... of others".

Clinical practice

Many participants indicated that they believe they became more effective in their interactions with clients, through a change in their capacity to manage their own emotions and distress tolerance, indicating an increase in mindfulness when working with patients: Responses included: "Easier to 'stay' with difficult patient and situations", "I find myself more present with clients and less reactionary ... to what clients are saying and feeling", "I'm more aware of my ego slipping into the room and the habit of thinking of responses to clients before they are even finishing talking", "Listening more and being quiet more".

As well as through changes in their capacity to cope with workload and related stressors; responses relating to this theme included: "More loving kindness to myself as expressed by offering myself more time between sessions ...", "Learned skills to relax when stressed and this has enabled me to do more i.e., to be more efficient in finishing my tasks", "Helped with work productivity and ability to slow down and cope with workload more effectively", "I have a tendency to jump to problem solving/change strategies when not mindful - using STOP has helped me STOP, validate, then work toward change".

Responses also indicated that participants are likely to utilise the knowledge from the intervention when providing psychoeducation to clients: "Using the breath to help with present moment connection to my clients", "I loved reflecting daily on some of the slogans ... using these within my work", "Helping clients connect more to their breath/sensations in their body", "encouraging it more with patients", "Realisation of the importance of sharing this practice with my client", "I have shared the STOP technique with pain clients and children and their families and they inform of finding it helpful."

Lastly, participants report a noteworthy change to the general relationship they have to their workplace as well as improvement to their workplace relationships: Responses relating to this theme included: "Less manifested irritation at other co-workers", "It has provided an opportunity to engage with colleagues in a different way and a greater sense of community (in a small way) which could grow ...", "I have been taking more initiative at work ... I feel more connected to my colleagues"

\section{Qualitative synthesis}

Overall, the major themes of this study, personal and clinical benefits of mindfulness, can be conceptualised in the context of improvement in two key interpersonal domains. The first is emotional reactivity. Several participants reported a marked reduction in emotional reactivity, which tended to be described as a sense of being able to better notice their own emotional reactions and reflect upon them without immediately responding. This ability to "stop and reflect" was frequently associated with the respondent's perceived improvement in their ability to listen and communicate. The second is listening and communication. Several participants described that they felt able to listen more deeply to clients 
during clinical work when emotionally distressing topics were being explored. Finally, a large proportion of participants described that they were able to communicate more effectively with family members and professional colleagues.

Several participants discussed how the reduction in emotional reactivity allowed them more time to reflect on responses, rather than reacting to the emotion of the situation. Subsequent changes in listening and communication were often directly related to the reduced emotional reactivity. As such, it appears that when clinicians are able to advance their mastery over their own emotional processes, they are able to more effectively attend to other's needs. This process is supported by the commonly used models of transference/counter-transference as well as mindful practice theory. ${ }^{46,47}$ Likewise, the combination of the above factors seems to have a commonly shared improvement on personal wellbeing through self-care, self-love, and increased connectedness to others.

\section{Discussion}

This study showed that a short, validated mindfulness course enhances components of, and overall mindfulness in mental-health clinicians, including psychiatrists. Critically, this finding was supported in the participants' subjective reporting of their experience, which provided more nuanced insight into how the benefits might manifest themselves, primarily through the emotional, communication, and relationship benefits of the program. This study provides a novel synthesis of quantitative and qualitative data relating to the impact of mindfulness training in the sample.

The findings from this study validate the results of previous research, indicating mindfulness is protective against burnout, and provides valuable insight into possible contributing factors. ${ }^{48}$ Participants reported that after the intervention they were able to listen to clients more deeply and communicate with patients and family more effectively, themes directly related to the observation and describing FFMQ subscales. Both observing and describing have been positively correlated with active listening and empathy, which in addition to the clinical and therapeutic benefits, are likely to be protective against burnout. $^{49,50}$

Non-reactivity contributes to emotional resilience by way of allowing one's emotions to occur without getting carried away. ${ }^{51}$ An increase in the non-reacting FFMQ subscale post MAPs was matched by participants' narrative reports of reduced emotional reactivity. Emotional reactivity is directly correlated with burnout. ${ }^{52}$ Thus, moderating this with mindfulness techniques provides a direct mechanism of burnout prevention.

There are a number of limitations to this study. Self-selection bias may have selected for those clinicians more 'psychologically minded'. Inherent limitations did not allow for blinding or control groups. Controlling for confounding variables such as the effects of medication or changes to the participants usual psychological treatment or social circumstances may have impacted the results. Furthermore, while the small sample size sufficed for the purpose of the pilot program, it will have almost certainly impacted on the statistical significance. High pre-test mean scores could have resulted in a ceiling effect, including awareness. Participants previous experience with mindfulness-based programs was not assessed and may have contributed to high pre-test FFMQ scores. Whilst no change in the mean PSS scores was observed, this could be explained by increased clinical demands and stressors in October, November, and December which is typically a busy time of the year for professionals in this field. Further studies can explore this through implementing numerous trials across the year.The COVID-19 pandemic has driven developments in the field of burnout prevention, ${ }^{53}$ which, may not be captured here due to the study being completed prior to the pandemic. Nevertheless, these findings may be useful for mental healthcare workers and organisations throughout the COVID-19 pandemic, to manage increasing stress and burnout. ${ }^{54}$

Despite the limitations, this study suggests personal and occupational benefits of mindfulness training in mentalhealth workers through enhanced resilience and ultimately prevention of burnout in mental health clinicians. To the best of the authors' knowledge, this is the first mixed-methods examination of a mindfulness intervention in the sample population. The subject would benefit from further research using a larger sample size, which would also provide valuable information to support the scalability and practicality of such an intervention.

\section{Data availability}

Underlying data

Figshare: 2016 Mindfulness Stats Complete.xlsx. https://doi.org/10.6084/m9.figshare.16566135.v1.55

This project contains the following underlying data:

- Amherst 2016 Mindfulness Stats Complete.xlsx: Participant demographic information

- PrePostPostRData_FFMQxlsx.xlsx: Pre- and post-test Five Facet Mindfulness Questionnaire scores 
- PrePostRawDataPSS.xlsx: Pre- and post-test Perceived Stress Scale scores

Figshare: De-identified qualitative responses. https://doi.org/10.6084/m9.figshare.16757029.v1. ${ }^{56}$

This project contains the following underlying data:

- Qual responses.pdf: Written responses to the two qualitative questions

Data are available under the terms of the Creative Commons Attribution 4.0 International license (CC-BY 4.0).

\section{Acknowledgements}

The authors wish to acknowledge Beth Wallace for delivering the Mindfulness Awareness Practices (MAPs) program.

1. Maslach C, Jackson SE, Leiter MP, et al.: Mas/ach burnout inventory. Palo Alto, CA: Consulting Psychologists Press; 1986.

2. Enshassi A, Al Swaity $E$, Arain $F$ : Investigating common causes of burnout in the construction industry. Int. J. Construction Project Manage. 2016; 8(1): 43.

3. Rada RE, Johnson-Leong C: Stress, burnout, anxiety and depression among dentists. J. Am. Dent. Assoc. 2004; 135(6): 788-94.

Publisher Full Text | PubMed Abstract

4. Taris TW, Bakker AB, Schaufeli WB, et al.: Job control and burnout across occupations. Psychol. Rep. 2005; 97(3): 955-61. PubMed Abstract | Publisher Full Text

5. Amoafo $\mathrm{E}$, Hanbali $\mathrm{N}$, Patel A, et al.: What are the significant factors associated with burnout in doctors?. Occup. Med. 2015; 65(2): 117-21.

PubMed Abstract | Publisher Full Text

6. Greenglass ER, Burke RJ, Fiksenbaum L: Workload and burnout in nurses. J. Community Appl. Soc. Psychol. 2001; 11(3): 211-215. Publisher Full Text

7. Lindblom KM, Linton SJ, Fedeli C, et al.: Burnout in the working population: relations to psychosocial work factors. Int. J. Behav. Med. 2006; 13(1): 51-9.

PubMed Abstract | Publisher Full Text

8. Rotenstein LS, Torre M, Ramos MA, et al.: Prevalence of burnout among physicians: a systematic review. JAMA. 2018; 320(11): $1131-50$.

PubMed Abstract | Publisher Full Text | Free Full Text

9. Embriaco N, Azoulay E, Barrau K, et al.: High level of burnout in intensivists: prevalence and associated factors. Am. J. Respir. Crit Care Med. 2007; 175(7): 686-92.

PubMed Abstract | Publisher Full Text

10. Kealy D, Halli P, Ogrodniczuk JS, et al.: Burnout among Canadian psychiatry residents: a national survey. Can. J. Psychiatry. 2016. 61(11): 732-6.

PubMed Abstract | Publisher Full Text | Free Full Text

11. Summers RF, Gorrindo T, Hwang S, et al.: Well-being, burnout, and depression among North American psychiatrists: the state of our profession. Am. J. Psychiatr. 2020; 177(10): 955-64.

Publisher Full Text

12. Lee FJ, Stewart M, Brown JB: Stress, burnout, and strategies for reducing them: what's the situation among Canadian family physicians?. Can. Fam. Physician. 2008; 54(2): 234-5 PubMed Abstract | Free Full Text

13. Murali $\mathrm{K}$, Banerjee $\mathrm{S}$ : Burnout in oncologists is a serious issue: what can we do about it?. Cancer Treat. Rev. 2018; 68: 55-61. PubMed Abstract | Publisher Full Text

14. Morse G, Salyers MP, Rollins AL, et al.: Burnout in mental health services: A review of the problem and its remediation. Adm. Policy Ment. Health Ment. Health Serv. Res. 2012; 39(5): 341-52. PubMed Abstract | Publisher Full Text | Free Full Text

15. Devebakan N: The relationship between burnout and perceived stress: A sample of healthcare workers. Psychiatry Behav. Sci. 2018; 8(4).
16. Hakanen JJ, Schaufeli WB, Ahola K: The Job Demands-Resources model: A three-year cross-lagged study of burnout, depression, commitment, and work engagement. Work \& Stress. 2008; 22(3): 224-41.

Publisher Full Text

17. Halbesleben JR, Rathert C: Linking physician burnout and patient outcomes: exploring the dyadic relationship between physicians and patients. Health Care Manag. Rev. 2008; 33(1): 29-39. PubMed Abstract | Publisher Full Text

18. Holmqvist $R$, Jeanneau M: Burnout and psychiatric staff's feelings towards patients. Psychiatry Res. 2006; 145(2-3): 207-13. PubMed Abstract | Publisher Full Text

19. Dewa CS, Jacobs P, et al.: An estimate of the cost of burnout on early retirement and reduction in clinical hours of practicing physicians in Canada. BMC Health Serv. Res. 2014; 14(1): 254. PubMed Abstract | Publisher Full Text | Free Full Text

20. McFarland DC, Hlubocky F, Riba M: Update on addressing mental health and burnout in physicians: What is the role for psychiatry?. Curr. Psychiatry Rep. 2019; 21(11): 108. PubMed Abstract | Publisher Full Text | Free Full Text

21. Bishop SR, Lau M, Shapiro S, et al.: Mindfulness: A proposed operational definition. Clin. Psychol. Sci. Pract. 2004; 11(3): 230-41.

22. Ludwig DS, Kabat-Zinn J: Mindfulness in medicine. JAMA. 2008; 300(11): 1350-2. PubMed Abstract | Publisher Full Text

23. Gold E, Smith A, Hopper I, et al.: Mindfulness-based stress reduction (MBSR) for primary school teachers. J. Child Fam. Stud. 2010; 19(2): 184-9. Publisher Full Text

24. Klatt MD, Buckworth J, Malarkey WB: Effects of low-dose mindfulness-based stress reduction (MBSR-Id) on working adults. Health Educ. Behav. 2009; 36(3): 601-14. PubMed Abstract | Publisher Full Text

25. Jain S, Shapiro SL, Swanick S, et al.: A randomized controlled trial of mindfulness meditation versus relaxation training: effects on distress, positive states of mind, rumination, and distraction. Ann. Behav. Med. 2007; 33(1): 11-21. PubMed Abstract | Publisher Full Text

26. Shapiro SL, Brown KW, Biegel GM: Teaching self-care to caregivers: Effects of mindfulness-based stress reduction on the mental health of therapists in training. Training and education in professional psychology. 2007; 1(2): 105-15. Publisher Full Text

27. Dobkin PL, Bernardi NF, Bagnis CI: Enhancing clinicians' wellbeing and patient-centered care through mindfulness. J. Contin. Educ. Health Prof. 2016; 36(1): 11-6. Publisher Full Text

28. Fortney L, Luchterhand C, Zakletskaia L, et al.: Abbreviated mindfulness intervention for job satisfaction, quality of life, and compassion in primary care clinicians: a pilot study. The Annals of Family Medicine. 2013; 11(5): 412-20. PubMed Abstract | Publisher Full Text | Free Full Text

29. Montero-Marin J, Tops M, Manzanera R, et al.: Mindfulness, resilience, and burnout subtypes in primary care physicians: the 
possible mediating role of positive and negative affect. Front. Psychol. 2015; 6: 1895.

30. Treadway MT, Lazar SW: The neurobiology of mindfulness. Clinical handbook of mindfulness. Springer; 2009; 45-57. Publisher Full Text

31. Almalki S: Integrating Quantitative and Qualitative Data in Mixed Methods Research--Challenges and Benefits.J. Educ. Learn. 2016; 5(3): 288-96 Publisher Full Text

32. Francis IJ, Johnston $M$, Robertson $C$, et al: What is an adequate sample size? Operationalising data saturation for theory-based interview studies. Psychol. Health. 2010; 25(10): 1229-45. PubMed Abstract | Publisher Full Text

33. Weiss RS: Learning from strangers: The art and method of qualitative interview studies. Simon and Schuster; 1995.

34. Black DS, O'Reilly GA, Olmstead R, et al.: Mindfulness meditation and improvement in sleep quality and daytime impairment among older adults with sleep disturbances: a randomized clinical trial. JAMA Intern. Med. 2015; 175(4): 494-501.

PubMed Abstract | Publisher Full Text | Free Full Text

35. Bower JE, Crosswell AD, Stanton AL, et al.: Mindfulness meditation for younger breast cancer survivors: a randomized controlled trial. Cancer. 2015; 121(8): 1231-40. PubMed Abstract | Publisher Full Text | Free Full Text

36. Baer RA, Smith GT, Hopkins J, et al.: Using self-report assessment methods to explore facets of mindfulness. Assessment. 2006 13(1): 27-45. PubMed Abstract | Publisher Full Text

37. Mattes J: Systematic review and meta-analysis of correlates of FFMQ mindfulness facets. Front. Psychol. 2019; 10: 2684. Publisher Full Text

38. Baer RA, Smith GT, Lykins E, et al.: Construct validity of the five facet mindfulness questionnaire in meditating and nonmeditating samples. Assessment. 2008; 15(3): 329-42. PubMed Abstract | Publisher Full Text

39. Cohen S, Kamarck T, Mermelstein R: A global measure of perceived stress. J. Health Soc. Behav. 1983; 24: 385-96. PubMed Abstract | Publisher Full Text

40. Ezzati A, Jiang J, Katz MJ, et al.: Validation of the Perceived Stress Scale in a community sample of older adults. Int. J. Geriatr. Psychiatry. 2014; 29(6): 645-52. PubMed Abstract | Publisher Full Text | Free Full Text

41. Cohen S, Kamarck T, Mermelstein R: Perceived stress scale. Measuring stress: A guide for health and social scientists. 1994; 10(2): 1-2.

42. Leung DY, Lam T-h, Chan SS: Three versions of Perceived Stress Scale: validation in a sample of Chinese cardiac patients who smoke. BMC Public Health. 2010; 10(1): 1-7. Publisher Full Text

43. Klein $\mathrm{EM}$, Brähler $\mathrm{E}$, Dreier $\mathrm{M}$, et al.: The German version of the Perceived Stress Scale-psychometric characteristics in a representative German community sample. BMC Psychiatry. 2016; 16(1): 1-10

Publisher Full Text

44. Braun V, Clarke V: Using thematic analysis in psychology. Qual. Res. Psychol. 2006; 3(2): 77-101.

Publisher Full Text

45. Nowell LS, Norris JM, White DE, et al.: Thematic analysis: Striving to meet the trustworthiness criteria. Int J Qual Methods. 2017; 16(1): 160940691773384

Publisher Full Text

46. Hayes JA, Gelso CJ, Hummel AM: Managing countertransference. Psychotherapy. 2011; 48(1): 88-97. PubMed Abstract | Publisher Full Text

47. Epstein RM: Mindful practice. JAMA. 1999; 282(9): 833-9. PubMed Abstract | Publisher Full Text

48. Goodman MJ, Schorling JB: A mindfulness course decreases burnout and improves well-being among healthcare providers. Int. J. Psych. Med. 2012; 43(2): 119-28. PubMed Abstract | Publisher Full Text

49. Jones SM, Bodie GD, Hughes SD: The impact of mindfulness on empathy, active listening, and perceived provisions of emotional support. Commun. Res. 2019; 46(6): 838-65. Publisher Full Text

50. Wagaman MA, Geiger JM, Shockley C, et al.: The role of empathy in burnout, compassion satisfaction, and secondary traumatic stress among social workers. Soc. Work. 2015; 60(3): 201-9. PubMed Abstract | Publisher Full Text

51. Gu J, Strauss $C$, Crane $C$, et al.: Examining the factor structure of the 39-item and 15-item versions of the Five Facet Mindfulness Questionnaire before and after mindfulness-based cognitive therapy for people with recurrent depression. Psychol. Assess. 2016; 28(7): 791-802.

PubMed Abstract | Publisher Full Text | Free Full Text

52. Keinan G, Melamed S: Personality characteristics and proneness to burnout: A study among internists. Stress Medicine. 1987; 3(4): 307-15.

Publisher Full Text

53. Sultana A, Sharma R, Hossain MM, et al.: Burnout among healthcare providers during COVID-19 pandemic: Challenges and evidence-based interventions. 2020.

54. Bradley $\mathrm{M}, \mathrm{Chahar} \mathrm{P}$ : Burnout of healthcare providers during Covid-19. Cleve. Clin. J. Med. 2020. Publisher Full Text

55. Watson T: 2016 Mindfulness Stats Complete.xIsx. figshare. Dataset. 2021. Publisher Full Text

56. Watson T: De-identified qualitative responses. figshare. Dataset. 2021.

Publisher Full Text 


\section{Open Peer Review}

\section{Current Peer Review Status:}

\section{Version 2}

Reviewer Report 23 March 2022

https://doi.org/10.5256/f1000research.120773.r121726

(C) 2022 Singaram V. This is an open access peer review report distributed under the terms of the Creative Commons Attribution License, which permits unrestricted use, distribution, and reproduction in any medium, provided the original work is properly cited.

\section{Veena S. Singaram}

School of Clinical Medicine, College of Health Sciences, University of KwaZulu-Natal, Durban, South Africa

Revisions are accepted. Submission is accepted for indexing.

Competing Interests: No competing interests were disclosed.

Reviewer Expertise: Health Professions Education

I confirm that I have read this submission and believe that I have an appropriate level of expertise to confirm that it is of an acceptable scientific standard.

\section{Version 1}

Reviewer Report 18 January 2022

https://doi.org/10.5256/f1000research.77400.r101856

(c) 2022 Singaram V. This is an open access peer review report distributed under the terms of the Creative Commons Attribution License, which permits unrestricted use, distribution, and reproduction in any medium, provided the original work is properly cited.

\section{Veena S. Singaram}

School of Clinical Medicine, College of Health Sciences, University of KwaZulu-Natal, Durban, South Africa

The authors pilot a mindfulness program (MAPs) with a group of health practitioners, making this study a highly topical and useful study since this group of practitioners (and others) has been 
found to undergo symptoms of burnout in healthcare settings. The authors found that the MAPs program had positive benefits for their participants, both personally and professionally.

The study may be outdated due to the outdated references and the absence of current findings. Recent findings on mindfulness and healthcare professional wellbeing literature have proliferated the field of healthcare professional wellbeing especially since the advent of COVID-19, causing the authors to miss out on current developments in the field of healthcare worker wellbeing and burnout. This absence is understandable since the data were collected before COVID-19 began. Perhaps a word or two from the authors to signal and explain what may appear anachronistic to some readers.

The authors also comment on the participants' high pre-test mean scores on the FFMQ and one wonders whether this may have been due to participants' previous experiences with mindfulnessbased programs and whether this does not partly explain the ceiling effect the authors speak about in their limitations.

Though the study design was apt, the literature review and discussion spoke of burnout, yet the assessment tested perceived stress scales (PSS). Burnout is not the same as stress, thus I would suggest explaining why PSS was used instead of a burnout inventory assessing the different components as described in the first line of the literature review. Or, I would suggest changing the literature review and conclusions commenting on stress levels instead of burnout.

In terms of details of qualitative analysis, the steps were not described enough to make it replicable. Please add a description of how exactly the data was collated and analysed.

Otherwise, the study is well put together, well written, and brings important attention to a group of healthcare practitioners professionally mandated to the mental well-being of others.

Is the work clearly and accurately presented and does it cite the current literature? Partly

Is the study design appropriate and is the work technically sound? Yes

Are sufficient details of methods and analysis provided to allow replication by others? Partly

If applicable, is the statistical analysis and its interpretation appropriate? I cannot comment. A qualified statistician is required.

Are all the source data underlying the results available to ensure full reproducibility? Yes

Are the conclusions drawn adequately supported by the results? Partly

Competing Interests: No competing interests were disclosed. 
Reviewer Expertise: Health Professions Education

\section{I confirm that I have read this submission and believe that I have an appropriate level of expertise to confirm that it is of an acceptable scientific standard, however I have significant reservations, as outlined above.}

Author Response 28 Jan 2022

Ashwin Varghese, Monash Health, Melbourne, Australia

We would like to thank the reviewers for their valuable input. We have addressed each comment in the revised submission. Specifically, we have expanded on the correlation between perceived stress and burnout, provided evidence to support this relationship, and explained why the PSS was used. We have provided a more thorough explanation of data collection and analysis. We have addressed the potential impacts of COVID-19 on the findings and made comments on the possible limitation of not knowing participants' previous experience with mindfulness.

Competing Interests: All authors report no competing interests.

Reviewer Report 22 November 2021

https://doi.org/10.5256/f1000research.77400.r100017

(C) 2021 Mottershead D. This is an open access peer review report distributed under the terms of the Creative Commons Attribution License, which permits unrestricted use, distribution, and reproduction in any medium, provided the original work is properly cited.

\section{Dr. Richard Mottershead}

Ras Al Khaimah College of Nursing, RAK Medical and Health Sciences University, Ras Al Khaimah, United Arab Emirates

Thank you for allowing me the opportunity to review this research article paper. I have read the article with interest and have the following comments to make:

This is a well-composed paper, which will be of interest to all healthcare practitioners working within health and social care settings. The authors are embedded within the practice and seek to make long-standing contributions to the research literature and clinical practice for health and social care professionals through the advent of mindfulness techniques which consequently improve clinical effectiveness. The paper proposes a holistic benefit of enhanced mindfulness from engagement with service users to family members. The necessity for health and social care works need to be within the moment, not only within work but in family life which necessitates positive mental health and well-being in all aspects of life.

It is an approach to care that advocates self-love and self-care which are often secondary outcomes for health and social care professionals. The authors identify the challenges of 
generalising results and snowballing techniques that may lead to attracting participants willing and able to engage and adopt highlighted practices but notwithstanding. The research is an engaging insight to adopting evidence-based practices to improve service delivery as well as staff mental well-being.

The paper will undoubtedly be followed by others and I wish the authors well. Their work seeks to establish long-term benefits to the health and well-being of practitioners that deserve support in maintaining and enhancing resilience which will of course benefit service user care and recovery.

Is the work clearly and accurately presented and does it cite the current literature?

Yes

Is the study design appropriate and is the work technically sound?

Yes

Are sufficient details of methods and analysis provided to allow replication by others? Yes

If applicable, is the statistical analysis and its interpretation appropriate?

Yes

Are all the source data underlying the results available to ensure full reproducibility? Yes

Are the conclusions drawn adequately supported by the results?

Yes

Competing Interests: No competing interests were disclosed.

Reviewer Expertise: I am a qualified researcher, mental health practitioner with experience of supporting health and social care practitioners mindfulness and mental well-being.

I confirm that I have read this submission and believe that I have an appropriate level of expertise to confirm that it is of an acceptable scientific standard. 
The benefits of publishing with F1000Research:

- Your article is published within days, with no editorial bias

- You can publish traditional articles, null/negative results, case reports, data notes and more

- The peer review process is transparent and collaborative

- Your article is indexed in PubMed after passing peer review

- Dedicated customer support at every stage

For pre-submission enquiries, contact research@f1000.com 